\title{
Controllable Encapsulation of "Clean" Metal Clusters within MOFs through Kinetic Modulation: Towards Advanced Heterogeneous Nanocatalysts
}

\author{
Hongli Liu, Lina Chang, Cuihua Bai, Liyu Chen, Rafael Luque, * and Yingwei Li*
}

\begin{abstract}
Surfactant-free tiny Pt clusters were successfully encapsulated within MOFs with controllable size and spatial distribution by a novel kinetically modulated one-step strategy. Our synthesis relies on the rational manipulation of the reduction rate of Pt ions and/or the growth rate of MOFs by using $\mathrm{H}_{2}$ as assistant reducing agent and/or acetic acid as $\mathrm{MOF}$ formation modulator. The as-prepared Pt@MOF core-shell composites exhibited exceedingly high activity and excellent selectivity in the oxidation of alcohols as a result of the ultrafine "clean" Pt clusters, as well as interesting molecular-sieving effects derived from the outer platinum-free MOF shell.
\end{abstract}

$M$ etal nanoparticles (MNPs) have received an enormous amount of research interest for decades owing to their unique properties and broad application in a variety of fields, especially in catalysis. ${ }^{[1]}$ In general, tiny particles with "clean" surfaces may offer a high surface-to-volume ratio and a great number of accessible active sites, and usually exhibit significantly improved catalytic performance as compared to their larger counterparts. ${ }^{[2]}$ However, free tiny MNPs often suffer from serious aggregation and fusion because of their increased surface energy accompanying a decrease in particle size, thus leading to the disappearance of their intriguing properties as well as difficulties associated with long-term storage, processing, and applications. Consequently, substantial effort has been devoted to the development of effective strategies to stabilize MNPs, ${ }^{[3]}$ and a promising approach is to disperse MNPs on various porous materials by enhancing the confinement effect of MNPs in the supports. ${ }^{[3 a-e]}$ The incorporation of tiny "clean" MNPs, especially in metal clusters (diameter $<2 \mathrm{~nm}$ ), within porous matrices with accurate control over their spatial

[*] Dr. H. Liu, L. Chang, C. Bai, L. Chen, Prof. Y. Li

School of Chemistry and Chemical Engineering

South China University of Technology

Guangzhou 510640 (China)

E-mail: liyw@scut.edu.cn

Prof. R. Luque

Departamento de Química Orgánica, Universidad de Córdoba Edif. Marie Curie, Ctra Nnal IVa Km 396, 14014, Córdoba (Spain) E-mail: q62alsor@uco.es

(iD) Supporting information and the ORCID identification number(s) for the author(s) of this article can be found under http://dx.doi.org/10. 1002/anie.201511009.

of (c) 2016 The Authors. Published by Wiley-VCH Verlag GmbH \& Co. $\mathrm{KGaA}$. This is an open access article under the terms of the Creative Commons Attribution Non-Commercial License, which permits use, distribution and reproduction in any medium, provided the original work is properly cited and is not used for commercial purposes. distribution is significant for practical applications, but exceedingly challenging.

Metal-organic frameworks (MOFs) are a unique class of porous materials synthesized by the adjustable assembly of metal ions and organic ligands; they are typically characterized by large surface areas, tunable and uniform pore structures, and interconnected three-dimensional cavities accessible through small pore windows. ${ }^{[4]}$ These characteristics make MOFs potentially useful for the spatial confinement of MNPs. Interaction/contact between the incorporated MNPs would be impeded, thus avoiding the agglomeration and detachment of the MNPs, whereas the smooth diffusion of reactants into and out of the MOFs would be guaranteed. More importantly, through the rational manipulation of the nucleation and growth of the MOFs and/or incorporated MNPs, it is expected that tiny MNPs could be encapsulated within crystalline MOFs with controlled spatial distribution.

Consequently, various methodologies have been developed to incorporate MNPs into MOFs, principally by either "ship-in-a-bottle"[5] or "bottle-around-a-ship"[6] approaches through a two-step or even multistep procedure. The former method is based on the reduction of a metal precursor doped in the already-formed MOFs and leads to tiny, naked MNPs. Nevertheless, some MNPs are inevitably deposited on the external surface of the MOF crystals because of the diffusion resistance between the external and internal MOF surfaces, thus resulting in a wide size range as well as random and unpredictable spatial distribution of MNPs. The latter, which involves the assembly of MOF precursors around prestabilized MNPs, is a straightforward and promising strategy to address the intrinsic problems facing the former approach. Regardless of the apparent advantages, however, certain capping agents are indispensable to restrict the aggregation of MNPs and facilitate MOF overgrowth on the MNPs. It is often difficult to fully remove these capping agents; thus, more complex and ill-defined interfaces between the MOF and MNPs result, as well as a physical barrier that hinders the access of reactants to the catalytically active MNPs. Moreover, it is still fairly difficult to control the inclusion of MNPs with sizes of less than $2 \mathrm{~nm}$ in the MOF matrix. Therefore, the development of a facile and effective approach for the incorporation of "naked" metal clusters (diameters $<2 \mathrm{~nm}$ ) within MOF matrices with accurate control of spatial distribution is imperative.

Recently, we demonstrated a facile one-step strategy to incorporate tiny Pt MNPs in MOF matrices by using $N, N$ dimethylformamide (DMF) as both a mild reductant for metal ions and a stabilizer for the formation of Pt NPs (Figure 1a). ${ }^{[6 c]}$ Unfortunately, owing to the much slower rate 


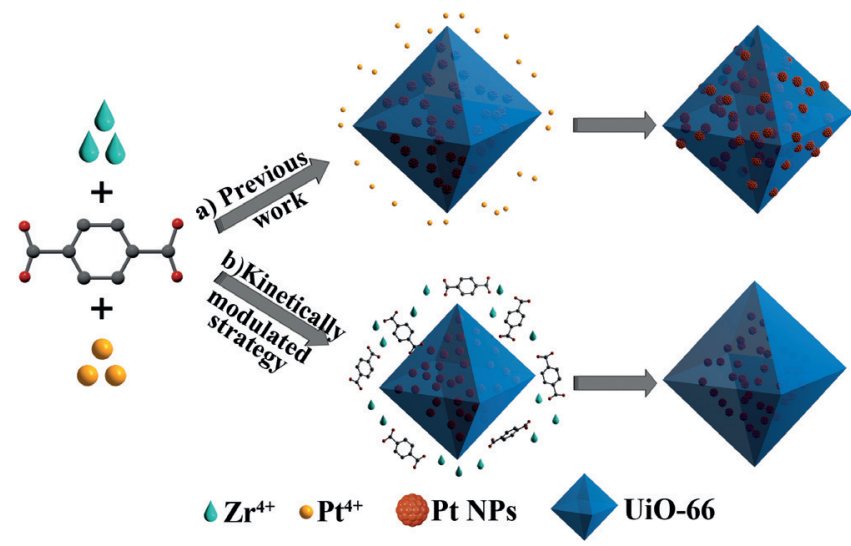

Figure 1. Incorporation of Pt NPs in MOFs through a) an in situ onestep strategy ${ }^{[6 c]}$ and b) a kinetically modulated in situ one-step strategy (this study).

of formation of Pt NPs as compared to the MOF, most of the Pt NPs were not well embedded within the MOF, thus leading to uneven and uncontrollable distribution of Pt NPs. Herein, we disclose a novel and efficient kinetically modulated onestep protocol for the controlled embedding of "clean" $\mathrm{Pt}$ clusters within MOF crystals. Control over the size and spatial distribution of Pt NPs is achieved by the use of acetic acid as a MOF-formation modulator and/or $\mathrm{H}_{2}$ as an assistant reducing agent (Figure $1 \mathrm{~b}$ ) to increase the reduction rate of $\mathrm{Pt}$ and/or decrease the growth rate of the MOF, respectively. The as-prepared Pt clusters embedded within MOFs exhibited high activity and stability in the aerobic oxidation of cinnamyl alcohol, as well as excellent size selectivity in olefin hydrogenation.

$\mathrm{UiO}-66,{ }^{[7]}$ a representative carboxylate-based MOF without any potential protecting groups for MNPs, features a large surface area and high physicochemical stability. We chose this MOF as an example to investigate the controllable incorporation of Pt clusters. Clearly different from the previously reported cumbersome methods, this one-step strategy involved the direct mixing of both the Pt (i.e., $\mathrm{H}_{2} \mathrm{PtCl}_{6}$ ) and MOF precursors in DMF in the present of acetic acid under a mixed $\mathrm{H}_{2}$ /air atmosphere. During the synthetic process, $\mathrm{H}_{2} \mathrm{PtCl}_{6}$ was first reduced to Pt clusters in a short time at the synthesis temperature of UiO-66 (i.e., $120^{\circ} \mathrm{C}$ ) with $\mathrm{H}_{2}$ and DMF as reducing agents. On the basis of the well-established hard and soft acid and base (HSAB) principle, ${ }^{[8]}$ the $\mathrm{Pt}$ clusters generated in situ might preferentially coordinate to the soft $\mathrm{C}-\mathrm{N}$ group of DMF, whereas the hard oxophilic $\mathrm{Zr}^{4+}$ cations for the MOF would show a preferential interaction with the hard $\mathrm{C}=\mathrm{O}$ group of DMF. This coordination would provide a "bridge" (i.e., DMF) between the MOF precursors and Pt nanoclusters, thus inducing preferential anisotropic growth of the MOF on the $\mathrm{Pt}$ surface rather than selfnucleation. Subsequently, MOF microcrystals would be produced and spontaneously grow around the surface of the Pt clusters, which are generated in situ by reduction. The formed MOF shell around the Pt clusters could serve as a protective layer to restrict the tiny Pt clusters from growing and aggregating during the synthesis. Hence, this strategy might not only avoid the use of a capping agent, but could also enable straightforward control over the particle size and spatial distribution of $\mathrm{Pt}$ by adjusting the reduction rate of $\mathrm{Pt}$ ions and/or the growth rate of the MOF.

Initially, we investigated the effect of the reduction rate of $\mathrm{Pt}$ ions on the encapsulation process. Considering the mild reduction ability of DMF, $\mathrm{H}_{2}$ was introduced into the synthetic system as a stronger reductant to promote the reduction of $\mathrm{Pt}$ ions. In a typical experiment, $\mathrm{ZrCl}_{4}, 1,4-$ benzene dicarboxylic acid, and acetic acid were dissolved in DMF and kept at $120^{\circ} \mathrm{C}$ for $24 \mathrm{~h}$ under $\mathrm{H}_{2}$ /air $(0: 1,1: 2,1: 1$, and $2: 1 \mathrm{v} / \mathrm{v}$ ). On completion of the reaction, the supernatant was dark gray in the absence of $\mathrm{H}_{2}$ (i.e., $\mathrm{H}_{2} /$ air $0: 1$ ), and the content of Pt doped on UiO-66 was only $0.7 \mathrm{wt} \%$ (ca. $35 \%$ of the total $\mathrm{Pt}$ added). With the introduction of $\mathrm{H}_{2}$, the reactionmixture supernatant was transparent and colorless, and analysis of the powder and supernatant by atomic absorption spectrometry (AAS) indicated that essentially all of the $\mathrm{Pt}$ was incorporated in the UiO-66 (2 wt \% ) regardless of the $\mathrm{H}_{2} /$ air ratio.

It has been documented that electron-beam irradiation during transmission electron microscopy (TEM) might destroy MOF structures and lead to agglomeration of the initially loaded small metal clusters. ${ }^{[9]}$ To minimize this effect, we used a low-intensity TEM electron beam $(<200 \mathrm{keV})$, thus maximizing the use of a defocused beam to limit the local beam current. TEM images were also recorded as quickly as possible to shorten the exposure time to the electron beam. We did not observe any significant changes in the size and distribution of metal clusters or the morphology of UiO-66 during our TEM measurements. TEM images showed highly monodisperse octahedral crystals composed of UiO-66 and Pt NPs for all the as-prepared Pt@UiO-66 materials (Figure 2). The results are consistent with the crystal morphology of the parent UiO-66, thus indicating that the incorporation of $\mathrm{Pt}$ did not affect MOF formation. The Pt NPs were unevenly dispersed and slight aggregation was observed in the absence of $\mathrm{H}_{2}$ (Figure $2 \mathrm{a}$ ). It was interesting to note that when $\mathrm{H}_{2}$ was introduced with a 1:2 $\mathrm{H}_{2}$ /air ratio, Pt clusters with a mean size of $(1.7 \pm 0.3) \mathrm{nm}$ were fully embedded into UiO-66 with an approximately $5 \mathrm{~nm}$ thick MNP-free MOF shell (Figure 2b; see also Figure $\mathrm{S} 1$ in the Supporting Information). As the $\mathrm{H}_{2}$ volume ratio was increased, the outer MNP-free UiO-66 shell gradually became thicker, whereas the size and dispersion of the Pt clusters remained almost unchanged (see Table S1 in the Supporting Information). Typical Pt dispersion in the materials was in the range of 53-71\%. Analysis of the cores by high-resolution TEM showed that the interplanar spacing of the particle lattice was about $0.226 \mathrm{~nm}$ (Figure $2 \mathrm{~g}$ ), which corresponds to the spacing of the (111) planes of facecentered cubic (fcc) Pt. HAADF-STEM imaging and corresponding EDX elemental mapping (Figure 2i) further demonstrated that $\mathrm{Pt}$ was homogeneously distributed throughout the MOF.

The characteristic XRD peaks of all of the above Pt@UiO-66 composites matched well with those of the parent UiO-66 (see Figure S2), thus suggesting that the incorporation of $\mathrm{Pt}$ did not affect the integrity of the MOF. Furthermore, no identifiable peaks associated with Pt NPs 

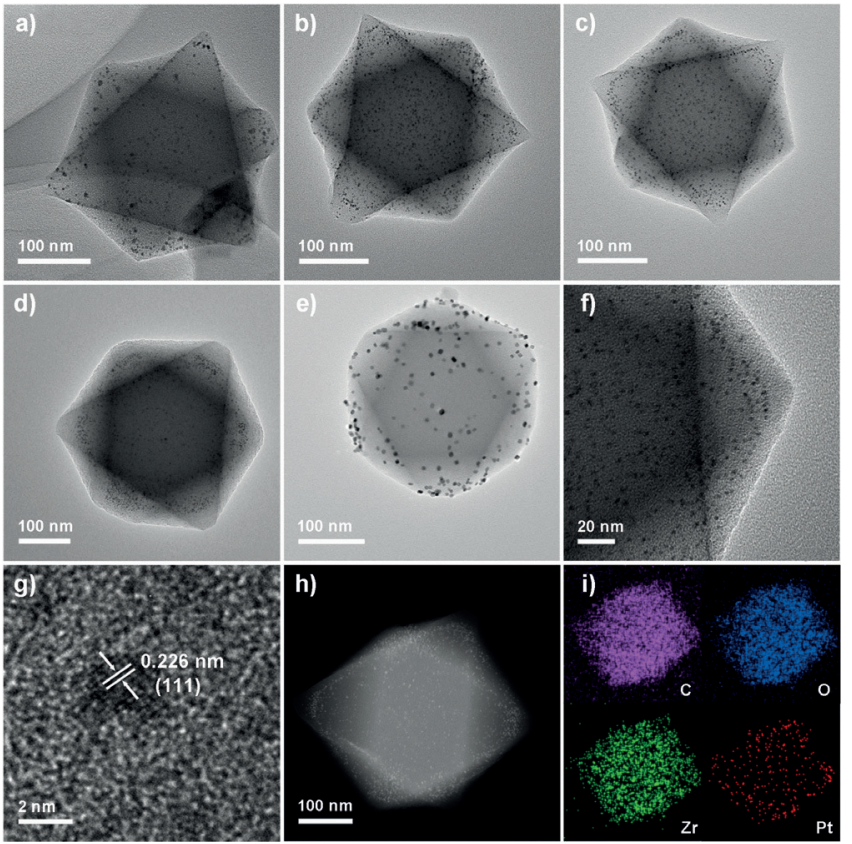

Figure 2. a-d,f,g) TEM images of Pt@UiO-66 nanocomposites prepared in the presence of $\mathrm{H}_{2}$ /air with a volume ratio of 0:1 (a), 1:2 (b), 1:1 (c, f, g), and 2:1 (d). e) TEM image of Pt/UiO-66 prepared by impregnation. h) HAADF-STEM image of Pt@UiO-66 prepared under 1:1 $\mathrm{H}_{2}$ /air, and i) the corresponding EDX elemental mapping.

were observed, possibly as a result of the low Pt content $(2 \mathrm{wt} \%)$ and/or the small particle size. The specific surface area and porosity of the samples were characterized by $\mathrm{N}_{2}$ physisorption at $77 \mathrm{~K}$. Pt@UiO-66 exhibited typical type I isotherms, with a steep increase in $\mathrm{N}_{2}$ uptake at relatively low pressures owing to the presence of microporosity (see Figure S3). Additionally, the pore-size distribution for all samples was similar and consistent with the cavity size of UiO-66.

The above analysis and experimental results suggested that the reduction rate of $\mathrm{Pt}$ was directly correlated with the extent to which the Pt clusters were loaded and encapsulated. We used a $\mathrm{UV} / \mathrm{Vis}$ spectrometer to gain insight into the conversion of $\mathrm{H}_{2} \mathrm{PtCl}_{6}$ over time in the solution in DMF and acetic acid under $\mathrm{H}_{2} /$ air at different ratios at $120^{\circ} \mathrm{C}$. The asprepared $\mathrm{H}_{2} \mathrm{PtCl}_{6} / \mathrm{DMF} /$ acetic acid solution ( $0 \mathrm{~min}$ ) displayed no appreciable difference in its UV/Vis absorption spectrum under different $\mathrm{H}_{2}$ /air atmospheres (see Figure S4). After $3 \mathrm{~h}$, a new shoulder peak at approximately $330 \mathrm{~nm}$ related to a ligand-to-metal electron transition from $\mathrm{N}$ to $\mathrm{Pt}$ cations in mono- and multinuclear $\mathrm{Pt}^{2+}$ complexes stabilized by $\mathrm{DMF}^{[10]}$ was observed in the absence of $\mathrm{H}_{2}$ (i.e., $\mathrm{H}_{2}$ /air 0:1, Figure S4b). Meanwhile, there was hardly any increase in the absorption intensity in the visible region, thus implying no formation of Pt NPs. ${ }^{[1]}$ Nevertheless, in the presence of $\mathrm{H}_{2}$, the characteristic absorption bands at approximately $330 \mathrm{~nm}$ corresponding to $\mathrm{Pt}^{2+}$ complexes were not observed (see Figure S4b), whereas the absorbance in the visible region clearly increased. Stronger absorption intensities were observed as the $\mathrm{H}_{2}$ volume ratio was increased, thus indicating a stronger reducing ability of $\mathrm{H}_{2}$ as compared to DMF.
The use of DMF as a reducing agent in the synthesis of MNPs normally involves the oxidation of DMF to carbamic acid; subsequently, the produced carbamic acid readily decomposes to dimethylamine at high temperatures during the reduction step. ${ }^{[12]}$ The amounts of the above products could not be detected in our synthesis, possibly as a result of the presence of hydrogen (decreasing DMF oxidation). Traces of such products (if produced) along with DMF could be readily removed by thorough washing and solvent exchange as well as vacuum heat treatment to produce clean-surface $\mathrm{Pt}$ NPs (see the Supporting Information for details). A further study indicated that trace amounts of amines did not significantly influence the preparation of Pt@UiO-66 materials (see Figure S5).

On the basis of hard and soft acid and base (HSAB) theory, DMF and possible traces of the intermediate $\mathrm{Me}_{2} \mathrm{NCOOH}$, which contain both hard $\mathrm{C}=\mathrm{O}$ or $\mathrm{COO}^{-}$ groups and soft $\mathrm{C}-\mathrm{N}$ groups, can probably coordinate simultaneously to hard oxophilic metal cations $\left(\mathrm{Zr}^{4+}\right)$ and soft nitrophilic Pt clusters. This feasible bridging effect between the UiO-66 precursors and Pt clusters offered the possibility of preferential nucleation and growth of UiO-66 around the Pt clusters formed in situ by reduction. The UiO66 crystals formed around the Pt clusters could then act as protective layers to confine the tiny Pt clusters and prevent their growth and aggregation during the synthesis. Accordingly, owing to the stronger reducing ability of $\mathrm{H}_{2}$ relative to that of DMF, the reduction time for $\mathrm{Pt}$ was effectively shortened as the amount of $\mathrm{H}_{2}$ increased (see Figure S4), thus leading to more rapid formation of the $\mathrm{Pt}$ cores as compared to UiO-66. The UiO-66 crystals grew around the continuously forming Pt clusters until all the Pt was depleted. Thus, the spatial position and distribution of the incorporated $\mathrm{Pt}$ clusters can be adjusted by varying the volume ratio of $\mathrm{H}_{2}$. However, in the absence of $\mathrm{H}_{2}$, because of the weak reducing ability of DMF, when the construction of UiO-66 was complete, most of the Pt cations had not been reduced and were still present in the reaction solution (Figure 1a). Consequently, these unencapsulated Pt species were partly deposited on the external surface of the UiO-66 crystals, and some were removed by centrifuging and washing, thus leading to uneven distribution and low loadings of Pt.

To further demonstrate the importance of confinement effects offered by the surrounding MOF shell, we prepared $2 \mathrm{wt} \% \mathrm{Pt} / \mathrm{UiO}-66$ by a traditional impregnation method. Because of the diffusion resistance between the external and internal surfaces of the MOF, most of the Pt NPs were located on the external surface of the UiO-66 crystals (Figure 2e). As expected, apparent aggregation and larger Pt NPs were observed as compared to the Pt@UiO-66 synthesized under similar conditions (Figure $2 \mathrm{a}-\mathrm{d}$ ).

To further confirm that Pt clusters were truly confined inside the UiO-66 crystals, we carried out liquid-phase hydrogenation of 1-hexene and tetraphenylethylene with the Pt@UiO-66 materials at $25^{\circ} \mathrm{C}$ and atmospheric $\mathrm{H}_{2}$ pressure. For comparison, we also tested the catalytic activity of Pt/UiO-66 prepared by impregnation. All Pt@UiO-66 samples showed the complete conversion of 1-hexene within $1 \mathrm{~h}$, and $\mathrm{Pt} / \mathrm{UiO}-66$ provided $73 \%$ conversion (Figure 3 ). The 


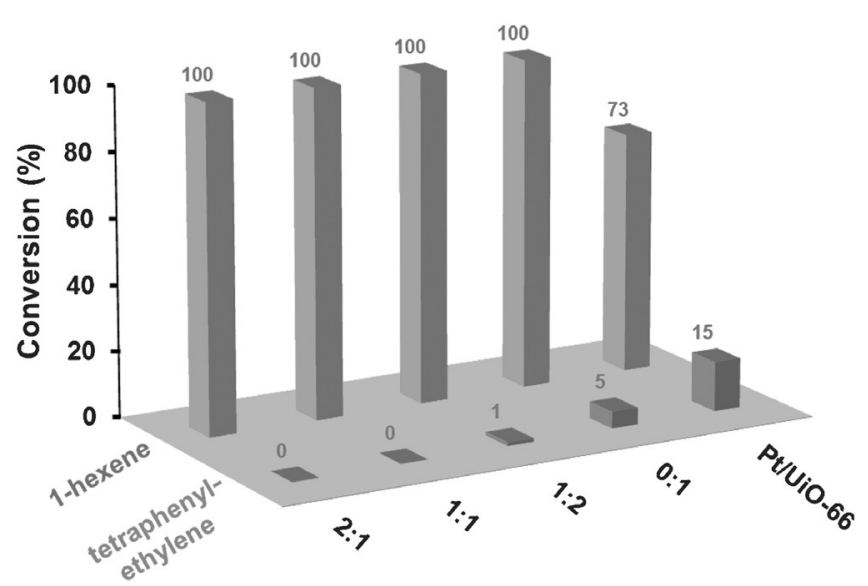

Figure 3. Size-selective hydrogenation of 1-hexene and tetraphenylethylene by Pt@UiO-66 prepared at different $\mathrm{H}_{2}$ /air ratios as compared to hydrogenation by $\mathrm{Pt} / \mathrm{UiO}-66$.

results indicated that there was no distinct diffusional resistance caused by the UiO-66 framework because the linear 1-hexene molecule was small enough (ca. $2.5 \AA$ in size) to diffuse into and out of the MOF cavities. When tetraphenylethylene with a larger size (ca. 6.7 $\AA$ ) was used, the Pt@UiO-66 prepared under 1:1 and 2:1 $\mathrm{H}_{2}$ /air showed no detectable activity; however, Pt@UiO-66 prepared under 0:1 and 1:2 $\mathrm{H}_{2}$ /air as well as $\mathrm{Pt} / \mathrm{UiO}-66$ still provided 5, 1 , and $15 \%$ conversion, respectively. These results could be ascribed to the molecular-sieving capability of the UiO-66 shell, through which the larger molecules could not diffuse to access the encapsulated $\mathrm{Pt}$ active sites. The results also demonstrated that essentially no Pt NPs were deposited on the external surface of the Pt@UiO-66 samples prepared under $\mathrm{H}_{2}$ /air with volume ratios of 1:1 and 2:1.

The benefit of UiO-66-encapsulated "clean" Pt clusters was demonstrated by the liquid-phase oxidation of cinnamyl alcohol, which is one of the most extensively investigated model reactions for assessing the catalytic activity of a catalyst. The reactions were carried out at $80^{\circ} \mathrm{C}$ and atmospheric pressure of $\mathrm{O}_{2}$ under base-free conditions. Initially, the oxidation was performed with the pristine UiO-66 to determine the baseline rate, and essentially no conversion was observed.

Subsequently, the Pt@UiO-66 materials prepared under different $\mathrm{H}_{2}$ /air atmospheres were employed for the reaction. Pt@UiO-66 (1:1 $\mathrm{H}_{2} /$ air) exhibited the highest catalytic activity and promoted the quantitative conversion of cinnamyl alcohol into cinnamyl aldehyde within $9 \mathrm{~h}$ (Figure 4). Pt@UiO-66 prepared under 2:1 $\mathrm{H}_{2} /$ air displayed slightly lower activity, probably owing to a lower molecular-diffusion rate caused by the thicker MOF shell. A longer time was required for complete conversion with the Pt@UiO-66 materials prepared under $0: 1$ and $1: 2 \mathrm{H}_{2}$ /air (15 and $10 \mathrm{~h}$, respectively). For comparison, we also prepared a Pt@UiO66-PVP material by a similar method to that described above, with the exception that a surfactant (i.e., polyvinylpyrrolidone, PVP) was added. As expected, the catalytic activity of Pt@UiO-66-PVP was significantly lower that that of Pt@UiO-66, possibly because residual PVP molecules on

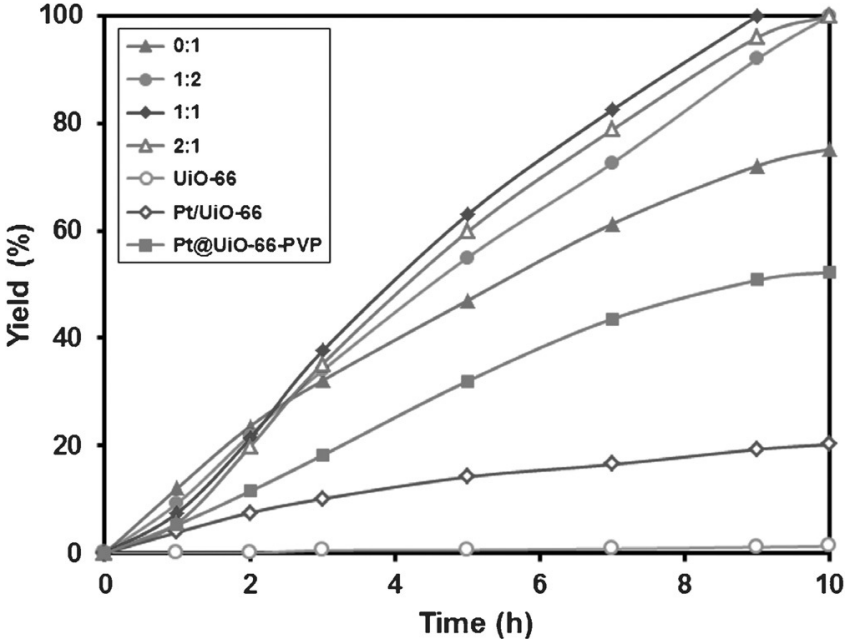

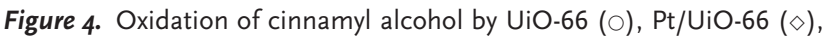
Pt@UiO-66-PVP (匹), and Pt@UiO-66 prepared under $\mathrm{H}_{2}$ /air with a volume ratio of 0:1 $(\mathbf{\Delta}), 1: 2(\bullet), 1: 1(\bullet)$, and 2:1 $(\triangle)$, respectively.

the Pt surface influenced its accessibility to reactants (Figure 4). Pt/UiO-66 was also tested in the oxidation of cinnamyl alcohol under identical conditions for comparison, and showed remarkably inferior activity. Considering that catalytic activity generally increases with decreasing MNP size, it is reasonable to believe that the lower catalytic activity of $\mathrm{Pt} / \mathrm{UiO}-66$ might be related to the size and distribution of Pt NPs (as indicated in Figure 2 and Table S1).

One crucial issue when working with supported metal catalysts is their stability and reusability, because leaching and aggregation of the metal active sites often occur during the course of reactions. Therefore, recycling and heterogeneity tests were performed on Pt@UiO-66 (1:1 $\mathrm{H}_{2} /$ air $)$ for the aerobic oxidation of cinnamyl alcohol. There was no appreciable reduction in activity and selectivity even after five runs, in accordance with PXRD (see Figure S2) and TEM observations (see Figure S7). In contrast, serious aggregation of Pt/ UiO-66 led to poor recycling performance (see Figure S7). The excellent stability and recyclability of Pt@UiO-66 can be attributed to the confinement effect offered by the outer UiO66 shell. A heterogeneity test indicated that Pt leaching was negligible and that the present alcohol oxidation occurred mostly on the heterogeneous Pt surface (see Figure S8).

Inspired by the above attractive properties, we attempted to modify the surfactant-free controlled encapsulation strategy by using acetic acid to modulate the nucleation rate of the MOF. Typically, materials were synthesized in a solution of $\mathrm{ZrCl}_{4}$, 1,4-benzene dicarboxylic acid, and various amounts of acetic acid $(0,60,90$, and 120 equiv with respect to $\mathrm{Zr})$ in $\mathrm{DMF}$ at $120^{\circ} \mathrm{C}$ under $\mathrm{H}_{2}$ /air (1:1). As expected, Pt clusters were encapsulated within MOF crystals, and a Pt-free MOF shell could also be distinguished as the amount of acetic acid increased (see Figures S10 and S11). These results demonstrated that the surfactant-free controlled encapsulation strategy was also possible by controlling the formation rate of MOFs.

In summary, we have demonstrated a novel kinetically modulated one-step strategy for the encapsulation within 
MOFs of surfactant-free tiny Pt clusters with controllable size and spatial distribution. This facile strategy was made possible by rational modulation of the reduction rate of $\mathrm{Pt}$ ions and/or the formation rate of MOF. The implementation of this method is also closely correlated with the use of the solvent DMF, which along with traces of its oxidization product $\mathrm{Me}_{2} \mathrm{NCOOH}$, probably act as a "bridge" to connect the MOF precursors and $\mathrm{Pt}$ clusters, thus inducing the anisotropic growth of MOF around the $\mathrm{Pt}$ surface. The synthesized Pt@UiO-66 core-shell catalysts exhibited high activity and excellent selectivity in the oxidation of alcohols, as well as molecular-sieving capability offered by the outer MOF shell. Furthermore, the catalysts were highly stable and readily reusable. Considering the wide use of DMF as a solvent in the synthesis of various MOFs as well as the HSAB principle, we speculate that this strategy could be extended to other types of MOFs (constructed from hard oxophilic metal cations, e.g., $\mathrm{Al}^{3+}, \mathrm{Ti}^{4+}$ ) encapsulating MNPs (e.g., $\left.\mathrm{Pd}, \mathrm{Au}\right)$. Such possibilities are currently under investigation in our laboratories. The developed one-step strategy might open new avenues for designing novel composite materials in which metal nanoparticles are encapsulated by MOFs for advanced catalytic applications.

\section{Acknowledgements}

This research was supported by the National NSF of China (21322606, 21436005, 21406075, and 21576095), FRFCU (2014ZB0004, 2015ZP002, and 2015PT004), Guangdong NSF (2013B090500027), and the China Postdoctoral Science Foundation (2014M550437 and 2015T80908).

Keywords: encapsulation - heterogeneous catalysis . metal clusters $\cdot$ metal-organic frameworks $\cdot$ platinum

How to cite: Angew. Chem. Int. Ed. 2016, 55, 5019-5023 Angew. Chem. 2016, 128, 5103-5107

[1] a) G. Chen, Y. Zhao, G. Fu, P. N. Duchesne, L. Gu, Y. Zheng, X Weng, M. Chen, P. Zhang, C.-W. Pao, J.-F. Lee, N. Zheng, Science 2014, 344, 495; b) J. A. Farmer, C. T. Campbell, Science 2010 , 329,933 ; c) H. Lee, S. E. Habas, G. A. Somorjai, P. D. Yang, $J$ Am. Chem. Soc. 2008, 130, 5406; d) C. Burda, X. Chen, R. Narayanan, M. A. El-Sayed, Chem. Rev. 2005, 105, 1025.

[2] a) Y. Lu, W. Chen, Chem. Soc. Rev. 2012, 41, 3594; b) K. Yamamoto, T. Imaoka, W. J. Chun, O. Enoki, H. Katoh, M Takenaga, A. Sonoi, Nat. Chem. 2009, 1, 397; c) P. Maity, S. Xie, M. Yamauchi, T. Tsukuda, Nanoscale 2012, 4, 4027; d) H. Qian, M. Zhu, Z. Wu, R. Jin, Acc. Chem. Res. 2012, 45, 1470.

[3] a) R. J. White, R. Luque, V. L. Budarin, J. H. Clark, D. J. Macquarrie, Chem. Soc. Rev. 2009, 38, 481; b) Ref. [1b]; c) F Zhang, X. Pan, Y. Hu, L. Yu, X. Chen, P. Jiang, H. Zhang, S. Deng, J. Zhang, T. B. Bolind, S. Zhang, Y. Huang, X. Bao, Proc. Natl. Acad. Sci. USA 2013, 110, 14861; d) P. Hu, J. V. Morabito, C.-K. Tsung, ACS Catal. 2014, 4, 4409; e) H. Tian, X. Li, L. Zeng, J. Gong, ACS Catal. 2015, 5, 4959; f) Y. Borodko, P. Ercius, V.
Pushkarev, C. Thompson, G. Somorjai, J. Phys. Chem. Lett. 2012, 3, 236; g) Z. Wu, J. Chen, R. Jin, Adv. Funct. Mater. 2011, 21, 177; h) Z. Bo, T. R. Eaton, J. R. Gallagher, C. P. Canlas, J. T. Miller, J. M. Notestein, Chem. Mater. 2015, 27, 1269.

[4] a) Z. Hu, B. J. Deibert, J. Li, Chem. Soc. Rev. 2014, 43, 5815; b) H. Furukawa, K. E. Cordova, M. O'Keeffe, O. M. Yaghi, Science 2013, 341, 1230444.

[5] a) D. Esken, S. Turner, O. I. Lebedev, G. V. Tendeloo, R. A. Fischer, Chem. Mater. 2010, 22, 6393; b) Q.-L. Zhu, J. Li, Q. Xu, J. Am. Chem. Soc. 2013, 135, 10210; c) L. Chen, H. Chen, R. Luque, Y. Li, Chem. Sci. 2014, 5, 3708; d) H. Liu, Y. Li, H. Jiang, C. Vargas, R. Luque, Chem. Commun. 2012, 48, 8431; e) T. Ishida, M. Nagaoka, T. Akita, M. Haruta, Chem. Eur. J. 2008, 14, 8456 ; f) Y. K. Park, S. B. Choi, H. J. Nam, D.-Y. Jung, H. C. Ahn, K. Choi, H. Furukawa, J. Kim, Chem. Commun. 2010, 46, 3086; g) A. Dhakshinamoorthy, H. Garcia, Chem. Soc. Rev. 2012, 41, 5262; h) J. Hermannsdörfer, M. Friedrich, N. Miyajima, R. Q. Albuquerque, S. Kümmel, R. Kempe, Angew. Chem. Int. Ed. 2012, 51, 11473; Angew. Chem. 2012, 124, 11640; i) A. Corma, H. Garcia, F. X. Llabres i Xamena, Chem. Rev. 2010, 110, 4606.

[6] a) Y. Liu, Z. Tang, Adv. Mater. 2013, 25, 5819; b) K. M. Choi, K. Na, G. A. Somorjai, O. M. Yaghi, J. Am. Chem. Soc. 2015, 137, 7810; c) H. Liu, L. Chang, L. Chen, Y. Li, J. Mater. Chem. A 2015, 3, 8028; d) W. Zhang, G. Lu, C. Cui, Y. Liu, S. Li, W. Yan, C. Xing, Y. R. Chi, Y. Yang, F. Huo, Adv. Mater. 2014, 26, 4056; e) M. Zhao, K. Deng, L. He, Y. Liu, G. Li, H. Zhao, Z. Tang, J. Am. Chem. Soc. 2014, 136, 1738; f) K. Na, K. M. Choi, O. M. Yaghi, G. A. Somorjai, Nano Lett. 2014, 14, 5979; g) L. He, Y. Liu, J. Liu, Y. Xiong, J. Zheng, Y. Liu, Z. Tang, Angew. Chem. Int. Ed. 2013, 52, 3741; Angew. Chem. 2013, 125, 3829; h) G. Lu, S. Li, Z. Guo, O. K. Farha, B. G. Hauser, X. Qi, Y. Wang, X. Wang, S. Han, X. Liu, J. S. DuChene, H. Zhang, Q. Zhang, X. Chen, J. Ma, S. C. J. Loo, W. D. Wei, Y. Yang, J. T. Hupp, F. Huo, Nat. Chem. 2012, 4, 310; i) T. Tsuruoka, H. Kawasaki, H. Nawafune, K. Akamatsu, ACS Appl. Mater. Interfaces 2011, 3, 3788.

[7] J. H. Cavka, S. Jakobsen, U. Olsbye, N. Guillou, C. Lamberti, S. Bordiga, K. P. Lillerud, J. Am. Chem. Soc. 2008, 130, 13850.

[8] a) R. G. Pearson, J. Chem. Educ. 1968, 45, 581; b) R. G. Pearson, J. Am. Chem. Soc. 1963, 85, 3533; c) A. Nag, M. V. Kovalenko, J. S. Lee, W. Liu, B. Spokoyny, D. V. Talapin, J. Am. Chem. Soc. 2011, 133, 10612.

[9] a) R. J. T. Houk, B. W. Jacobs, F. E. Gabaly, N. N. Chang, A. A. Talin, D. D. Graham, S. D. House, I. M. Robertson, M. D. Allendorf, Nano Lett. 2009, 9, 3413; b) B. W. Jacobs, R. J. T. Houk, M. R. Anstey, S. D. House, I. M. Robertson, A. A. Talin, M. D. Allendorf, Chem. Sci. 2011, 2, 411.

[10] a) K. Sakai, Y. Tanaka, Y. Tsuchiya, K. Hirata, T. Tsubomura, S. Iijima, A. Bhattachardjee, J. Am. Chem. Soc. 1998, 120, 8366; b) T. N. Fedotova, G. G. Aleksandrov, G. N. Kuznetsova, Russ. J. Inorg. Chem. 2008, 53, 372; c) Y. Borodko, C. M. Thompson, W. Huang, H. B. Yildiz, H. Frei, G. A. Somorjai, J. Phys. Chem. C 2011, 115, 4757.

[11] a) D. G. Duff, P. P. Edwards, B. F. G. Johnson, J. Phys. Chem. 1995, 99, 15934; b) N. Maeda, T. Eitoku, Y. Ikezoe, K. Katayama, Phys. Chem. Chem. Phys. 2012, 14, 200.

[12] a) I. Pastoriza-Santos, L. M. Liz-Marán, Adv. Funct. Mater. 2009, 19, 679; b) J. Yu, S. Schreiner, L. Vaska, Inorg. Chim. Acta 1990, $170,145$.

Received: November 27, 2015

Published online: March 11, 2016 